ISSN: 0212-0267

DOI: http://dx.doi.org/IO.I420I/hedu2OI53435737I

\title{
LOS SISTEMAS ESTANDARIZADOS \\ DE EVALUACIÓN EN CHILE: \\ PARTICIPACIÓN DE MARIO LEYTON SOTO Y ERIKA HIMMEL KÖNIG
}

\section{Standardized assessment systems in Chile: share of Mario Leyton Soto and Erika Himmel König}

\author{
Jaime CAiceo Escudero \\ Universidad de Santiago de Chile \\ Correo e: jcaiceo@hotmail.com
}

Recepción: I2 de mayo de 20I4. Envío a informantes: 30 de mayo de 2014.

Fecha de aceptación definitiva: 26 de junio de 2014

Resumen: La evaluación educacional, como ciencia de la educación, comenzó a desarrollarse y a aplicarse en Chile sólo a partir de los sesenta del siglo pasado. En efecto, varios educadores chilenos fueron a doctorarse a Estados Unidos de Norteamérica en esas materias; entre ellos se destacan Mario Leyton y Erika Himmel, quienes se especializaron en currículum y evaluación principalmente, a través de los planteamientos de Tyler y Bloom. Por lo mismo, ambos participaron activamente en la introducción en el país de dos sistemas estandarizados de medición: (I) El sistema de selección para el ingreso a la universidad, creando una prueba estandarizada, la Prueba de Aptitud Académica -PAA-, la cual se estableció a partir de 1967 y se mantuvo hasta el 2002 y desde el 2003 a la fecha se cambió a Prueba de Selección Universitaria -PSU-; inicialmente la PAA contenía sólo las pruebas de aptitud verbal y matemática; posteriormente se agregaron pruebas de conocimientos específicos (en matemática, biología, física, química y ciencias sociales). (2) La medición de los logros educacionales del sistema escolar, tanto en la educación básica ${ }^{1}$ como media ${ }^{2}$, a partir de la década del 70 con el denominado inicialmente Programa de Evaluación del Rendimiento Escolar -PER- y, posteriormente, con el Sistema de Medición de la Calidad de la Enseñanza -SIMCE-, el cual se aplica desde 1988 hasta hoy, a nivel

Corresponde a la educación primaria de otros países.

Corresponde a la educación secundaria de otros países. 
nacional y en diversos niveles del sistema escolar: cuartos básicos, octavos básicos y segundos medios, especialmente con pruebas de lenguaje y comunicación y matemática. En ambas iniciativas participaron directamente Mario Leyton y Erika Himmel; el primero en puestos claves del Ministerio de Educación y la segunda como académica de la Universidad de Chile y de la Universidad Católica de Chile. Por lo mismo, ambos han sido reconocidos por el Estado con el Premio Nacional de Ciencias de la Educación en el 2009 y 20II, respectivamente.

Palabras Clave: evaluación educacional; medición; pruebas estandarizadas; ingreso a la universidad (PAA-PSU); evaluación sistema escolar (PER-SIMCE).

Aвstract: Educational evaluation, as the science of education, began to be developed and applied in Chile only starting from the sixties of the last century. Indeed, several Chilean educators were to his $\mathrm{PhD}$ to United States of America in these areas; among them are Mario Leyton and Erika Himmel, who specialized in curriculum and evaluation, through the approaches of Tyler and Bloom. Therefore, both were actively involved in the introduction into the country of two measurement systems: (I) selection for admission to the University, creating a standardized test, the academic aptitude test-PAA- which was established from 1967 and remained until 2002 and from 2003 to date was changed to the university selection test-PSU-; initially the PAA contained only verbal and mathematical aptitude tests; knowledge tests were subsequently added specific (in mathematics, biology, physics, chemistry and Sciences social). (2) the measurement of achievements educational system school, both in basic education ${ }^{3}$ and medium ${ }^{4}$ from the I970s with the called initially program of evaluation of educational achievement-PER-and, subsequently, with the system of measurement of the quality of the teaching -SIMCE-, which applies from 1988 until today, at the national level and at various levels of the school system: $4 .^{\circ}$ Basic, $8 .^{\circ}$ Basic and $2 .^{\circ}$ Medium, especially with tests of language and communication and mathematics. Both initiatives were directly involved Mario Leyton and Erika Himmel; the first to key positions in the Ministry of Education and the second as academic of the University of Chile and the Catholic University of Chile. Therefore, both have been recognized by the State with the National Prize of Sciences of Education in 2009 and 20II, respectively.

KEY wORDs: Educational assessment; Measurement; Standardized tests; Admission to the University (PAA-PSU); School system evaluation (PER-SIMCE).

\section{Introducción}

$\mathrm{L}$

a evaluación educacional en Chile se ha estado valorizando en los últimos años por diversas razones, tanto académicas como de otro orden; sin embargo, lo que se quiere destacar en esta Ponencia dice relación con la importancia que el Ministerio de Educación le ha dado a esta área del saber

3 It corresponds to the primary education of other countries.

4 It corresponds to the secondary education of other countries. 
LOS SISTEMAS ESTANDARIZADOS DE EVALUACIÓN EN CHILE: PARTICIPACIÓN DE MARIO LEYTON SOTO Y ERIKA HIMMEL KÖNIG JAIME CAICEO ESCUDERO

educacional al otorgarle el Premio Nacional en Ciencias de la Educación a dos destacados educadores ligados a este ámbito de las Ciencias de la Educación; en efecto, en el 2009 fue galardonado Mario Leyton Soto y en el 2ori Erika Himmel König; el primero especialista en currículum, pero exalumno de Benjamín Bloom y quien colaboró activamente en el gobierno de Eduardo Frei Montalva desde sus diferentes cargos ministeriales, especialmente como subsecretario de Educación, para cambiar el bachillerato por la PAAs; la segunda, por ser una de las mentoras de la PAA, a partir de $1967^{6}$.

El objetivo de este artículo es dar a conocer el rol que le cupo a ambas personalidades educacionales en la introducción en Chile de los sistemas de evaluación, tanto a nivel del sistema escolar como de ingreso a la universidad. La metodología utilizada es la propia de los estudios históricos, recurriendo a fuentes primarias (entrevistas a testigos presenciales y documentos legales) y secundarias (documentos e investigaciones realizadas). El sustento teórico de la evaluación se basará especialmente en los planteamientos de Benjamín Bloom. Las conclusiones apuntan a que ambos educadores fueron claves en la incorporación de los sistemas de medición estandarizados en Chile.

Este artículo está basado en las investigaciones que el autor viene realizando desde 1996 a la fecha acerca de la vida, pensamiento y acción de los Premios $\mathrm{Na}$ cionales de Educación en Chile (I979-20II), tanto en la Universidad Metropolitana de Ciencias de la Educación como en la Universidad de Santiago de Chile.

\section{El Sistema de Selección Universitario y el Inicio de las Pruebas estandarizadas en Chile}

En relación al origen del sistema de selección universitario no existe unanimidad en los especialistas al respecto; es así como Ernesto y Paulina Schiefelbein ${ }^{8}$ señalan que este sistema existe desde mediados del siglo xIx; en cambio, Donoso ${ }^{9}$ indica que éste existe desde I931; seguramente la diferencia está en la fecha de formalización del mismo. En efecto, los primeros hacen relación al bachillerato

- Cfr. Sмiтh, Francisco: Mario Leyton y su aporte al desarrollo educacional de Chile en la segunda mitad del siglo XX, Tesis para optar al Grado de Magíster en Educación, Mención Gestión Educacional, Santiago de Chile, Universidad de Santiago de Chile, 20I2, I43 pp.

6 Cfr. Carrasco, Carmen Gloria: Rol de Erika Himmel en el Proceso de Gestación de la Prueba de Aptitud Académica como Instrumento de Evaluación para Ingresar a las Universidades Chilenas, Tesis para optar al Grado de Magíster en Educación, Mención Currículum y Evaluación, Santiago de Chile, Universidad de Santiago de Chile, 2013, ıoo pp.

Producto de esas investigaciones se ha publicado un primer tomo ( $c f r$. CaIceo, Jaime: Pensamiento y Acción Educativos en Chile a partir de los Premios Nacionales de Ciencias de la Educación, tomo I: Premiados 1979-1993, Santiago de Chile, Universidad de Los Lagos, 2012, 256 pp.

8 Schiefelbein, Ernesto y Schiefelbein, Paulina: «Evolución de los Procesos de Evaluación del Sistema Educativo 1950-2008», Revista Iberoamericana de Evaluación Educativa, I (I) (2008), pp. 45-50, extraído de http://www.rinace.net/riee/números/voli-numi/art3.pdf. Consultado el in de julio de 2012 .

9 Donoso, Sebastián: «La Reforma Educacional y el Sistema de Selección de Alumnos a las Universidades: Impactos y Cambios Demandados», Estudios Pedagógicos, Universidad Austral de Valdivia, n. ${ }^{\circ} 24$ (1998), pp. 7-30. 
LOS SISTEMAS ESTANDARIZADOS DE EVALUACIÓN EN CHILE: PARTICIPACIÓN DE MARIO LEYTON SOTO Y ERIKA HIMMEL KÖNIG JAIME CAICEO ESCUDERO

como el primer instrumento creado para ingresar a la Universidad de Chile en la misma ley de fundación de la misma en $\mathbf{1 8 4 2}$, ratificado posteriormente en la ley de educación secundaria y superior de i879; el segundo, en cambio, se refiere al momento en que se determina el Reglamento y Organización de bachillerato como sistema de admisión a las universidades existentes a la fecha, a través del DFL n. ${ }^{\circ} 28 \mathrm{O}^{\mathrm{Io}}$; el mencionado decreto en su art. 43 establece:

La Facultad de Filosofía y Ciencias de la Educación otorgará el grado de Bachiller en Humanidades, con mención en ramos o grupos de ramos del Programa de Estudios Secundarios, a los alumnos que estén en posesión de la Licencia Secundaria y que rindan satisfactoriamente las pruebas especiales que determine un Reglamento que dictará el Consejo Universitario. El grado de Bachiller en Humanidades será necesario para incorporarse a las Escuelas universitarias y para obtener los grados y títulos que la Universidad confiere ${ }^{\text {II }}$.

Sin embargo, todos los especialistas concuerdan en que a lo largo de la historia de las universidades chilenas han existido tres sistemas de selección para ingresar a las mismas: el mencionado bachillerato que existió hasta 1966, la Prueba de Aptitud Académica -PAA- que se aplicó entre 1967 y el 2002 y la Prueba de Selección Universitaria -PSU- que existe desde el 2003 a la fecha.

\section{I. El bachillerato (I842 o I93I a I966)}

Este instrumento existió con las diferencias planteadas anteriormente en cuanto a su origen, administrado por la Universidad de Chile en sus inicios, pero posteriormente la Universidad Técnica del Estado estableció un bachillerato industrial, diferente al bachillerato en humanidades de la Universidad de Chile y lo suyo hicieron las Universidades Católica de Valparaíso y Católica de Chile. Consistía en un conjunto de pruebas que se aplicaban a los egresados de la educación secundaria, las cuales evaluaban el dominio de diversas materias de ese nivel de enseñanza; eran pruebas con respuestas abiertas, lo cual complicaba la objetividad de los resultados en la medida que fue creciendo el número de alumnos que las rendían y como, además, había diferentes bachilleratos, ello impedía que existiera un sistema uniforme de ingreso a las universidades.

Las pruebas del bachillerato a lo largo de su extensa historia tuvieron muy pocas variaciones, salvo la mantención o no del latín; las pruebas constantes eran de

- La Universidad de Chile, fundada en I842; la Universidad Católica de Chile, fundada en I888; la Universidad de Concepción, fundada en 1920; la Universidad Técnica Federico Santa María, fundada en 1926, y la Universidad Católica de Valparaíso, fundada en I928. Tanto la Escuela de Artes y Oficios, fundada en 1849 y base de la Universidad Técnica del Estado, fundada en 1947, y como las Escuelas Preceptoras o Normales fundadas a partir de i842, no estaban afectas a este sistema de selección. Cfr. CAICEO, Jaime: «Educación Superior en Chile y su Internacionalización», Revista HISTEDBR On-line, ISSN: 1676-2584, Universidade Estadual de Campinas, Brasil, n. ${ }^{\circ} 38$, junhio (20IO), pp. I2/23: http:// www.histedbr.fae.unicamp.br/revista/edicoes/38/index.html. Consultado el 5 de septiembre de 2010 .

in Biblioteca del Congreso Nacional: DFl 280: Estatuto Orgánico Universitario de la Universidad de Chile, Santiago de Chile, I931, 3I de mayo, ı pp. 
LOS SISTEMAS ESTANDARIZADOS DE EVALUACIÓN EN CHILE: PARTICIPACIÓN DE MARIO LEYTON SOTO Y ERIKA HIMMEL KÖNIG JAIME CAICEO ESCUDERO

lenguaje materno, un idioma extranjero, historia y geografía de Chile y la prueba específica según a la carrera que postulara el alumno.

Los críticos del sistema lo empezaron a catalogar de elitista en la medida que se fue masificando la educación secundaria, especialmente a partir del inicio de la segunda mitad del siglo pasado; según Schiefelbein y Schiefelbein ${ }^{12}$ «sólo un $5 \%$ a ı\% de cada grupo de edad rendía esa prueba». A su vez, un estudio de Erika Grassau ${ }^{13}$ concluyó que había escasa relación predictiva entre el puntaje del bachillerato y el rendimiento académico de los alumnos en la universidad; la misma académica en 1966 señaló que, a pesar de los cambios que se le fueron introduciendo al bachillerato, el sistema ya no tenía la racionalidad inicial, pues la complejidad era muy grande porque había muchos postulantes, tanto de liceos como de colegios particulares y postulaban a carreras de ocho universidades diferentes e, incluso, tanto la Universidad de Chile como la Universidad Técnica del Estado poseían Sedes Regionales, agravando más la situación ${ }^{14}$. Por su parte, empeoró más la situación el tema de la corrección de las pruebas con respuesta abierta a un número cada vez más masivo de alumnos, los cuales podían, a su vez, elegir temas al azar. Finalmente, Rossetti y Manzi is indican que el sistema del bachillerato dividía a la población estudiantil en dos grupos muy diferentes: los egresados de la educación secundaria, de clase media o superior, podían dar la prueba de ingreso a la universidad y postular a la misma; los de clase baja sólo podían egresar de la educación primaria y luego ingresar al mundo laboral. Todo lo indicado hizo necesario buscar un sistema diferente, que fuera uniforme y único, poniéndose fin a este instrumento de evaluación en 1966.

La tesis de pregrado de Erika Himmel, dirigida por su maestra Grassau, versó sobre la capacidad predictiva del bachillerato. Sobre sus conclusiones, señala que «el instrumento no tenía la capacidad de anticipar cómo le iba a ir al alumno en su primer año de estudios superiores ${ }^{16}$; también recuerda otra investigación: «Estábamos al tanto por otro estudio que se había hecho en ingeniería de la Universidad de Chile, que había pruebas de física que las corrigieron siete profesores distintos y las calificaciones fueron de uno a siete. Entonces era absolutamente poco confiable el resultado $»^{17}$.

En todo el contexto anterior, en I966, tal como ya se indicó, se decreta el fin del bachillerato y el inicio de la Prueba de Aptitud Académica. El subsecretario de Educación, quien toma la decisión política junto al ministro Gómez, era Mario Leyton Soto ${ }^{18}$. «En el fondo la P.A.A. nació en el contexto de democratización

I2 Schiefelbein, Ernesto y Schiefelbein, Paulina: op. cit.

13 Grassau, Erika: Análisis estadístico de las pruebas del Bachillerato de la Universidad de Chile, Santiago de Chile, Universidad de Chile, 1956, 165 pp.

${ }_{14}$ Grassau, Erika: Los exámenes de Admisión a la Universidad, Santiago de Chile, Instituto de Investigaciones Estadísticas de la Universidad de Chile, 1966, is $\mathrm{pp}$.

is Rossetti, Josefina y Manzi, Jorge: «La evaluación educacional en Chile: Antecedentes y desarrollo reciente», Persona y Sociedad, XVIII (3) (2004), pp. 22I-242 en http://www.personaysociedad. $\mathrm{cl} / \mathrm{la}$-evaluacion-educacional-en-chile-antecedentes-y-desarrollo-reciente/. Consultado el 2 de julio de 2012.

${ }_{16}$ Himmel, Erika: Entrevista, Santiago de Chile, in de enero de 2013.

${ }_{17}$ Ibidem.

18 Mario Leyton no sólo tomó la decisión política junto a Juan Gómez Millas, sino que también, como se indicó anteriormente, había participado en el Seminario que se realizó en 1963 sobre 
LOS SISTEMAS ESTANDARIZADOS DE EVALUACIÓN EN CHILE: PARTICIPACIÓN DE MARIO LEYTON SOTO Y ERIKA HIMMEL KÖNIG JAIME CAICEO ESCUDERO

del sistema de educación chileno» ${ }^{19}$, que fue el gran objetivo de la Reforma Educacional de 1965 .

\subsection{Prueba de Aptitud Académica-PAA- (1967-2002)}

Erika Grassau, destacada académica de la Universidad de Chile, era experta en estadística y, tal como se indicó precedentemente, realizó varias investigaciones acerca de la predicción entre el bachillerato y el rendimiento de los alumnos en la universidad; además, había participado en la creación del Instituto de Investigaciones Estadísticas -el actual DEMRE, Departamento de Evaluación, Medición y Registro Educacional-, junto a Irma Salas ${ }^{20}$. Erika Himmel, por su parte, fue su alumna y comenzó a trabajar con ella a fines de la década de los 50, teniendo una activa participación en las investigaciones sobre alternativas de ingreso a la universidad dirigidas por su maestra Grassau.

Por otra parte, el nuevo gobierno, encabezado por Eduardo Frei Montalva, iniciaba la más importante Reforma Educacional del siglo xx en 1965; su ministro de Educación fue Juan Gómez Millas, quien en 1963 era rector de la Universidad de Chile y había colaborado para que Mario Leyton Soto, antiguo alumno suyo en el Instituto Pedagógico de la misma universidad, fuera a estudiar un Máster en Educación a la Universidad de Chicago en Estados Unidos. Maestro y discípulo mantenían correspondencia en ese período y el rector Gómez fue a Estados Unidos y pasó a ver al maestrando Leyton; allí conversó sobre rendimiento escolar y evaluación a propósito del cambio que deseaba hacerse en el proceso de ingreso a la universidad; le comentó que se haría un Seminario sobre esa temática y lo invitó a colaborar en el mismo con su experiencia académica en el país del norte, explicando nuevos tipos de instrumentos para cambiar el bachillerato, prueba existente en ese momento.

El Seminario, al cual fue invitado a participar Mario Leyton, fue dirigido por Óscar Vera Lamperein ${ }^{21}$; éste fue financiado por la Fundación Ford, realizado en la Facultad de Filosofía y Educación de la Universidad de Chile y organizado por

Rendimiento Escolar y Evaluación; a su vez, en 1964 participó, junto a Erika Grassau en el Demre: «Allí estuve trabajando junto con Erika Himmel y todo el equipo, perfeccionando el desarrollo de este instrumento (P.A.A.) y otros más» (LeYTon, Mario: Entrevista, Santiago de Chile, 8 de enero de 20I3).

19 Ibidem.

20 Esta importante educadora chilena se había doctorado en la Universidad de Columbia en Nueva York en 1930; la misma institución en que obtuvo su Máster la profesora Himmel 28 años después. La tesis de la Dra. Salas versó sobre La Composición Socio-económica del Estudiante Secundario en Chile, iniciando con ella diversas investigaciones empíricas en Chile (cfr. CAICEO, Jaime et al.: Filósofos y Educadores. El Pensar Chileno en el Siglo XX, Santiago de Chile, Facultad de Educación de la Pontificia Universidad Católica de Chile, I992, 343 pp.); Erika Grasaau y Erika Himmel continuarán en la segunda mitad del siglo xx.

${ }^{21}$ El año anterior (1962) había dirigido la Comisión de Planificación Educacional que había creado el gobierno de Jorge Alessandri para responder a los requerimientos que el país había contraído en 1956 en Uruguay en el contexto de la planificación educacional de los países latinoamericanos, propuesta que había sido lanzada y defendida por la Unesco en las Naciones Unidas, avalada por el gobierno del presidente Kennedy; estaba en el ámbito de lo que se denominó la Alianza para el Progreso del asesinado presidente norteamericano (LeYTon, Mario: op. cit.). 
LOS SISTEMAS ESTANDARIZADOS DE EVALUACIÓN EN CHILE: PARTICIPACIÓN DE MARIO LEYTON SOTO Y ERIKA HIMMEL KÖNIG JAIME CAICEO ESCUDERO

el Instituto de Investigaciones Estadísticas. A tal Seminario, con una duración de dos meses, asistieron 30 personas, entre chilenos y extranjeros, y una de ellas fue Erika Himmel. Los principales temas tratados fueron la elaboración de instrumentos de evaluación desde el punto de vista pedagógico, definición, selección y formulación de ítemes ${ }^{22}$.

En esa misma época había regresado Erika Grassau de un curso en Estados Unidos, quien venía con la idea de crear la Prueba de Aptitud Académica, adaptando la prueba SAT -Scholastic Aptitude Test o Scholastic Assessment' ${ }^{23}$ Test-, prueba estandarizada que se usaba frecuentemente para seleccionar el ingreso a la educación superior en aquel país. Coincide con ello el hecho que el Educational Testing Service -en donde estuvo Erika Grassau- decidiera expandirse hacia América Latina, para lo cual abrió una sede en Puerto Rico, traduciendo la prueba SAT al castellano; se deseaba iniciar pruebas piloto en varios países, incluido Chile. Sin embargo, el precio era muy alto para el país y se desechó la idea y se decidió preparar una propia. Por lo mismo, se comenzo a aplicar pruebas experimentales, tanto a estudiantes de primer año universitario como del último de la educación media -especialmente a estudiantes de las Sedes Regionales de la propia Universidad de Chile-, dando origen de esta forma a la PAA, la cual fue inscrita como propiedad intelectual de la Universidad Pública el 22 de octubre de I966, bajo el registro n. ${ }^{\circ}$ 30.965. Acorde con lo anterior, el Consejo Universitario de la Universidad de Chile, en sesión del 7 de septiembre de ese mismo año, aprobó aplicar la mencionada prueba para seleccionar a los alumnos que postularan a todas sus carreras e invitó a las siete universidades restantes para que utilizaran el mismo instrumento. Lo anterior permitió que el mismo año se promulgara la Ley n. ${ }^{\circ}$ I6.526, con fecha I3 de septiembre ${ }^{24}$, la cual suprimió «el bachillerato como requisito de ingreso a las Escuelas Universitarias dependientes de la Universidad de Chile, Universidad Técnica del Estado y de las Universidades reconocidas por el Estado» (art. n. ${ }^{\circ}$ I) e instauró la licencia media como fase terminal del sistema escolar (art. n. ${ }^{\circ}$ 2, inciso 3) y como requisito legal para ingresar a la universidad (art. 2, inciso 4). Todo lo anterior permitió que el in de enero de 1967 se aplicara por primera vez la Prueba de Aptitud Académica de la Universidad de Chile en todo el país y asumida por todas las demás universidades.

La supresión del bachillerato, que era un grado académico, fue reemplazado por un sistema -la PAA- que no otorgaba ningún grado ${ }^{25}$.

La Prueba de Aptitud Académica-PAA- se estructuró definitivamente en el Departamento de Evaluación, Medición y Registro Educacional -DEMRE- de

22 Leyton, Mario: op. cit.

23 Este término significa que se puede obtener información objetiva acerca de cómo actuarían las personas en diferentes circunstancias y tareas, pero sin formarse un juicio valorativo de las personas. Erika Himmel señala que ello no es apropiado en la evaluación educacional, pues la evaluación de los aprendizajes requiere del componente valorativo para poder mejorar el proceso de aprendizajeenseñanza, ya que es tanto para el alumno, como para el profesor ( $c f r$. CARRAsco, Carmen Gloria: op. cit.).

${ }^{24}$ Biblioteca del Congreso Nacional: Ley n. ${ }^{\circ}$ I6.526: Suprime el Bachillerato, Santiago de Chile, 1966, I3 de septiembre, 3 pp.

${ }_{25}$ Soto, Fredy: Historia de la Educación Chilena, Santiago de Chile, CPEIP, 2000, I83 pp. 
LOS SISTEMAS ESTANDARIZADOS DE EVALUACIÓN EN CHILE: PARTICIPACIÓN DE MARIO LEYTON SOTO Y ERIKA HIMMEL KÖNIG JAIME CAICEO ESCUDERO

la Universidad de Chile, sistema al cual adhirieron todas las otras universidades existentes en 1967 ( 8 en total, con sus respectivas sedes). El objetivo de esta prueba era uniformar y aunar criterios comunes en el proceso de selección universitaria; entregaba un ranking de puntajes entre 200 y 800 puntos aproximadamente; cada universidad determinaba los puntajes mínimos para ingresar a cada una de sus carreras. Contenía tres pruebas comunes: aptitud verbal, aptitud matemática e historia de Chile, transformada en obligatoria a partir de 1984 y pruebas específicas de biología, física, química, matemática y ciencias sociales. El requisito para rendir estas pruebas era haber egresado de la educación media. En la corrección para evitar el azar se descontaba una pregunta correcta por cuatro incorrectas.

Al interpretar lo relatado, queda claro que la antigua concepción de estado docente, acuñada durante el siglo xix con la Universidad de Chile a la cabeza como organismo rector, se mantenía aún en la segunda mitad del siglo xx.

Las mayores ventajas del nuevo sistema frente al bachillerato consistía en (i) que medía, en su parte común y general, habilidades o aptitudes y no tanto conocimientos; lo positivo de ello estaba en que alumnos sin los conocimientos adecuados en la enseñanza media, pero que tenían habilidades, no quedaban fuera de la posibilidad de ingresar a la universidad; las pruebas específicas sí que medían conocimientos de los contenidos programáticos de la educación media; y (ii) los resultados masivos permitían comparar los rendimientos con diferentes variables, tales como sexo, edad, origen socioeconómico, capital cultural de la familia, procedencia del alumno en cuanto establecimiento y dependencia administrativa (pública o privada); estos datos favorecían la toma de decisiones en el ámbito educativo $^{26}$. La desventaja, por su parte, se encontraba en que las mediciones al ser masivas eran estandarizadas, lo cual no consideraba la situación particular de cada alumno.

En todo el proceso descrito participó Erika Himmel como parte del equipo diseñador de la Prueba de Aptitud Académica, dirigido por Erika Grassau y compuesto, entre otras personas, por Lucy Bianchi, Teresa Segure, Luis Menke y Cristina Rodríguez. Al respecto, recuerda:

Y empezamos a desarrollar la prueba gradualmente durante cinco años, con apoyo de un buen número de universidades como la Pontificia Universidad Católica de Chile, la Pontificia Universidad Católica de Valparaíso, la Universidad de Concepción, y así empezamos, poco a poco, a desarrollarla, validarla, a ver si ésta sí tenía un buen pronóstico acerca de cómo le iba a los niños en la universidad... éramos un grupo chico, muy cohesionado, y trabajábamos bien artesanalmente ${ }^{27}$.

En sus primeras aplicaciones los alumnos respondían en tarjetas con hoyos, usando lápiz Faber n. ${ }^{\circ}$ 2, las cuales se leían en una máquina óptica donada por la Fundación Ford.

26 Cfr. Carrasco, Carmen Gloria: op. cit.

27 Himmel, Erika: op. cit. 
LOS SISTEMAS ESTANDARIZADOS DE EVALUACIÓN EN CHILE: PARTICIPACIÓN DE MARIO LEYTON SOTO Y ERIKA HIMMEL KÖNIG JAIME CAICEO ESCUDERO

\subsection{Prueba de Selección Universitaria-PSU- (2003 a la fecha)}

Por su parte, a partir de i98I en adelante, el Consejo de Rectores había establecido varias comisiones para que analizaran el sistema de ingreso a la universidad, debido a la profunda transformación que la educación superior tuvo a contar de ese año, debido a la reforma realizada a la educación superior en el país, estableciendo nuevas universidades públicas a partir de las Sedes Regionales de las dos universidades estatales existentes a la fecha, manteniendo las seis universidades privadas existentes (tres católicas y tres laicas) y permitiendo la creación de nuevas universidades privadas (DFL n. ${ }^{\circ}$ I); se estableció, a su vez, la posibilidad de crear Institutos Profesionales (DFL n. ${ }^{\circ}$ ) y Centros de Formación Técnica (DFL n. ${ }^{\circ} 24{ }^{28}$. Sin embargo, las críticas no fueron comparables a las que realizó la Comisión que funcionó en el 200o; en efecto, las razones esgrimidas para eliminar el bachillerato ya no eran válidas, especialmente las que indicaban que las aptitudes en las personas eran estables, y que, por lo tanto, no eran susceptibles de ser entrenadas y que, además, se distribuían en forma equivalente en los diferentes grupos socioeconómicos. Lo anterior, había quedado superado debido al avance de la psicología y las investigaciones posteriores a 1967, tanto a nivel nacional como internacional, las cuales indicaban que las personas podían ser entrenadas con mejores resultados y que había diferencias en las aptitudes entre los diferentes grupos socioeconómicos ${ }^{29}$.

En cuanto a la participación de Erika Himmel en la Psu, Cristián Cox, educador que estuvo más de io años en los gobiernos de la Concertación liderando los cambios curriculares, recuerda que el ministro de Educación, José Pablo Arellano (1996-2000), le solicitó en 1996 que revisara el tema de la PAA, a propósito de la Reforma en el sistema escolar que se inició ese año; formó una Comisión e invitó a Erika Himmel a participar en ella; esta Comisión funcionó entre 1999 y el 2000 y estaba compuesta por académicos de la Universidad de Chile y de la Pontificia Universidad Católica de Chile; el Informe Final fue presentado el 22 de noviembre del 200o. Textualmente señala:

Fue allí cuando a mí se me reveló uno de los rasgos que la hacen la persona excepcional que es. Y es que ella estaba reformando un instrumento que era la P.A.A., que había ordenado la selección universitaria por décadas, que ella misma había ayudado a formular en los 60 , y respecto al cual interviene 30 años después con mirada fresca, contextualizada y acorde a los nuevos requerimientos. En general, no ocurre mucho eso en las construcciones institucionales en educación, es decir, que un actor sea lo suficientemente reconocido por tan largo tiempo y vigente. E intervenir en dos momentos separados por 30 años. Eso es absolutamente excepcional y aún lo es más, que alguien vuelva sobre sus propias obras con otra mirada y no termine casado con su propia obra que es lo que le ocurre al $95 \%$ de los mortales, que defienden lo que hicieron, sin visión de superación por los tiempos y las circunstancias ${ }^{30}$.

Caiceo, Jaime: «Educación Superior en Chile y su Internacionalización», op. cit.

Schiefelbein, Ernesto y Schiefelbein, Paulina: op. cit.

3. Cox, Cristián: Entrevista, Santiago de Chile, 2I de diciembre de 2012. 
LOS SISTEMAS ESTANDARIZADOS DE EVALUACIÓN EN CHILE: PARTICIPACIÓN DE MARIO LEYTON SOTO Y ERIKA HIMMEL KÖNIG JAIME CAICEO ESCUDERO

Un participante de esa Comisión, Jorge Manzi, director del MideUC y exalumno de Erika Himmel, recuerda que tal Comisión, compuesta por académicos de la Universidad de Chile y de la Pontificia Universidad Católica de Chile, era presidida por Cristián Cox en conjunto con Erika Himmel, y entre algunos otros miembros recuerda a Erika Grassau y Cristina Rodríguez; en total eran I3. A su vez, el propio Manzi, junto a David Bravo de la Universidad de Chile, presentó un Proyecto de Investigación para reformular la PAA e invitaron a Erika Himmel. «Fue un gesto súper interesante de alguien que había estado desarrollando un instrumento y que luego estuvo dispuesta para cambiarlo... era una persona abierta al cambio. El proyecto lo presentamos durante el año 2000, se aprobó el 200r; se llamaba Proyecto Fondes del sies que luego derivó en la Prueba de Selección Universitaria $-\mathrm{PSU} \rightarrow \gg^{3 \mathrm{I}}$.

Ello derivó en un intenso debate público que llevó a que finalmente en el 2002 se aprobara el Sistema de Selección Universitario elaborado por el DEMRE de la Universidad de Chile; su aplicación comenzó a partir del 2003. De esta forma, se reemplazaron las ocho pruebas existentes (tres comunes obligatorias y cinco específicas optativas) por cuatro pruebas obligatorias: lenguaje, matemática, ciencias sociales y ciencias (con opciones de biología, física y química). A su vez, el énfasis de las nuevas pruebas debiera estar más relacionado con los contenidos programáticos vigentes, especialmente en la parte verbal y matemática; es decir, las nuevas pruebas debían focalizarse en los Contenidos Mínimos Obligatorios derivados de la LOCE -Ley Orgánica Constitucional de la Enseñanza- de i99o.

Esta situación trajo de inmediato dos posiciones contrapuestas: (i) la encabezada por Raczynski y Salinas ${ }^{32}$ y por $\operatorname{Cox}^{33}$, quienes afirmaban que era mejor preparar las pruebas con los contenidos habituales que los alumnos conocían y ello no implicaba abandonar las aptitudes, ya que ambos conceptos -contenidos y aptitudes- no se contraponen al elaborar un instrumento de medición, ya que esta última considera habilidades cognitivas o aptitudes aplicadas a determinados contenidos; (ii) uno de los educadores más críticos de esta nueva prueba es el Premio Nacional de Educación 2009, Mario Leyton Soto, quien señala:

Si uno analiza la PSu, está más orientada a medir contenido que habilidades, o sea aprendizaje de contenidos y no formativos ni significativos. Lo que se requiere que se logren procesos mentales superiores de meta-cognición, de que los alumnos sean capaces de pensar por sí mismos, evaluar sus propios trabajos, ser capaces de evaluar cómo van avanzando en su aprendizaje propiamente tal, mirarse a sí mismos, de poder relacionarse socialmente en forma efectiva con los pares, poder integrarse a la sociedad de forma armónica, positiva; sería convertirse en profesionales capaces de desarrollar trabajos múltiples que sólo se da cuando hay una sólida formación general. Hoy día la gente cambia cuatro, cinco veces de trabajo

31 Manzi, Jorge: Entrevista, Santiago de Chile, 2I de diciembre de 2012.

32 RacZYNSKI, Dagmar y SAlinas, Daniel: «Aportes y Desafíos en la Evaluación de Políticas y Programas de Mejoramiento Educativo. Reflexiones desde Nuestra Práctica de Evaluación en Chile», Revista Iberoamericana de Evaluación Educativa, I (I) (2008), pp. 79-92 en http://www. rinace.net/riee/numeros/voli-numi/art6.pdf. Consultado el is de julio de 2012.

33 Cox, Cristián: Politicas Educacionales en el Cambio de Siglo, Santiago de Chile, Editorial Universitaria, 2003, 7II pp. 
LOS SISTEMAS ESTANDARIZADOS DE EVALUACIÓN EN CHILE: PARTICIPACIÓN DE MARIO LEYTON SOTO Y ERIKA HIMMEL KÖNIG JAIME CAICEO ESCUDERO

en la vida, es casi lo normal, y para poder hacer eso, la educación tiene que entregarle instrumentos que son procesos mentales potentes que le permitan adecuarse a situaciones nuevas con rapidez. Eso es lo que llamamos procesos formativos, en términos de entregarles herramientas a los educandos en el contexto de velar por el desarrollo de esa persona, primero como persona fundamentalmente ${ }^{34}$.

Por lo expuesto, desde su génesis, la psu ha provocado fuertes debates, especialmente en aspectos sociopolíticos, puesto que se afirma que la nueva Prueba aumentaría la brecha entre alumnos egresados de establecimientos privados y los provenientes de la educación pública o municipal y más aún, los egresados de establecimientos técnicos quedarían definitivamente fuera del ingreso a la universidad, pues en su currículum prevalecen las asignaturas profesionales y no humanista-científicas. Precisamente, ésta ha sido una de las banderas de lucha de los movimientos estudiantiles del 2006 y desde el 201 ir en adelante; sin embargo, el cuestionamiento ha sido a la PSU en su conjunto.

Con la controversia que esta prueba ha generado, ha quedado claro que nuestros dos Premios Nacionales están en posturas contrapuestas: Himmel apoyándola y Leyton criticándola.

\section{El Programa de Evaluación del Rendimiento Escolar -PER-y Sistema de Medición de la Calidad de la Educación -SIMCE-}

El sistema escolar chileno comenzó a ser medido a partir de la Reforma de I965; en efecto, entre 1968 y i97i se realizó una Prueba Nacional a los alumnos de $8 .^{\circ}$ Año Básico con el objeto de medir los logros de aprendizaje ${ }^{35}$. De acuerdo a los antecedentes entregados por Mario Leyton, en el Instituto de Investigaciones Estadísticas de la Universidad de Chile, mientras se probaba el instrumento denominado PAA - por el equipo dirigido por Erika Grassau e integrado por Erika Himmel, entre otras-, también se realizaron pruebas de ensayo de lo que sería el Programa de Evaluación de Rendimiento Escolar -PER-; textualmente indica: "Yo mismo escribí un documento, sobre la creación de un sistema nacional de evaluación que implicaba el desarrollo de pruebas nacionales en $4 .^{\circ}$ y $8 .^{\circ}$ básico. Es decir, nosotros hicimos el diseño del sistema; en el Instituto siguieron construyendo el instrumento» ${ }^{36}$.

Formalmente comenzó a aplicarse el PER a partir de 1982, tanto en $4 .^{\circ}$ como en $8 .^{\circ}$ básico; su diseño definitivo lo ejecutó el Departamento de Investigación y Tecnología de la Universidad Católica -DiCTUC-, gracias a un Convenio con el Ministerio de Educación; en el mismo intervino Erika Himmel, en calidad de coordinadora técnica, junto a otros investigadores, «con el objetivo de institucionalizar diversas iniciativas en el ámbito de la evaluación que venían desarrollándose en

34 LeYTon, Mario: op. cit.

35 Bravo, David: «SIMCE: Pasado, Presente y Futuro del Sistema Nacional de Evaluación», Estudios Públicos, Santiago de Chile, n. ${ }^{\circ}$ I23 (20II), pp. I89-2II.

36 Leyton, Mario: op. cit. 
LOS SISTEMAS ESTANDARIZADOS DE EVALUACIÓN EN CHILE: PARTICIPACIÓN DE MARIO LEYTON SOTO Y ERIKA HIMMEL KÖNIG JAIME CAICEO ESCUDERO

nuestro país desde los años sesenta» ${ }^{37}$; al respecto, nuestra Premio Nacional recuerda: «Cuando nosotros partimos hace mucho tiempo atrás, te estoy hablando de su precursor, el PER, los resultados de éste se informaban por grupo curso, que era bastante más detallado que el actual SIMCE, donde es por nivel $\star^{38}$.

Entre 1985 y 1986 el Centro de Perfeccionamiento, Experimentación e Investigaciones Pedagógicas -CPEIP- elaboró el Sistema de Evaluación de la Calidad de la Educación -SECE-, con el objeto de analizar los datos generados por el PER ${ }^{39}$.

Finalmente, en 1988, en la propia Universidad Católica de Chile, un grupo de investigadores, «liderado por Erika Himmel» $»^{40}$ puso en práctica el SIMCE, a fin de evaluar el rendimiento escolar y la calidad de la educación impartida a nivel de $4 .{ }^{\circ}$ y $8{ }^{\circ}$ básico de todos los establecimientos educacionales del país. Con ello se perseguía: (i) Proporcionar asistencia al Ministerio de Educación en su tarea normativa y en la supervisión del sistema. (ii) Brindar apoyo a las autoridades regionales y locales en la supervisión, además de prestarles apoyo técnico. (iii) Estimar la calidad de la educación impartida en cada establecimiento educacional; comparar dichos resultados, buscar los factores explicativos y evaluar los resultados de los programas pedagógicos. (iv) Orientar la actividad de perfeccionamiento docente, las acciones de supervisión y la asignación de recursos ${ }^{4}$. A partir de 1992, el SIMCE pasó a ser controlado por el Ministerio de Educación, a fin de dar cumplimiento a la LOCE (Ley Orgánica Constitucional de Enseñanza), del io de marzo de I990, la cual estableció que «Corresponderá al Ministerio de Educación Pública diseñar los instrumentos que permitan el establecimiento de un sistema para la evaluación periódica, tanto de la enseñanza básica como media, de los objetivos fundamentales y contenidos mínimos de esos niveles» ${ }^{42}$.

Con el retorno a la democracia, el énfasis educativo estuvo centrado en la calidad, puesto que la demanda educativa prácticamente estaba toda cubierta en la educación básica y media. Por lo mismo, el Ministerio de Educación intensificó los sistemas de evaluación al sistema escolar, aumentó la extensión y frecuencia del $\operatorname{SIMCE}^{43}$ e integró al país a pruebas internacionales, tales como

37 El Mercurio: «Erika Himmel, Premio Nacional de Educación 20II», I4 de septiembre de 20II, C6, obtenido el in de enero de 20I3, desde http://diario.elmercurio.com/detalle/index. asp?id $=\{9484 \mathrm{fcod}-859 \mathrm{a}-4 \mathrm{~b} 4 \mathrm{f}-\mathrm{a} 6 \mathrm{I} 7-25 \mathrm{ac} 8 \mathrm{aa} 2 \mathrm{I} 2 \mathrm{~d} 4\}$.

${ }_{38}$ Himmel, Erika: op. cit.

39 Román, Marcela: «Usos Alternativos del simce: Padres, Directores y Docentes», en Documentos $n .{ }^{\circ}$ 5. Santiago de Chile: CIDE, I999, $89 \mathrm{pp}$.

${ }^{4}$ El Mercurio: op. cit.

${ }^{41}$ Bravo, David: op. cit.

${ }^{42}$ Ministerio de Educación: Decreto Ley $n .^{\circ} 40$-Loce-, Santiago de Chile, 1990, is pp.

43 Hasta el año 2005 , la aplicación de las pruebas se alternó entre $4 .^{\circ}$ Básico, $8 .^{\circ}$ Básico y $2 .{ }^{\circ}$ Medio; en el año 2006, se comenzó a evaluar todos los años a los estudiantes de $4 .^{\circ}$ Básico y se alternó entre los niveles de $8 .^{\circ}$ Básico y $2 .^{\circ}$ Medio. El año 2008 se realizó una prueba muestral de Escritura en $4 .^{\circ}$ Básico y el año 2010 se incorporaron tanto la evaluación en $3 .^{\circ}$ Medio del sector Idioma Extranjero, Inglés, como una primera evaluación muestral de Educación Física en $8 .^{\circ}$ Básico. En el presente año 2013 habrá Pruebas SIMCE en $2 .^{\circ}$ Básico (Comprensión de Lectura), $4 .^{\circ}$ Básico (Comprensión de Lectura, Matemática y Ciencias Naturales), 6. ${ }^{\circ}$ Básico (Comprensión de Lectura, Escritura y Matemática; los estudiantes del país con Discapacidad Sensorial rendirán las pruebas de Comprensión de Lectura y Matemática), $8 .^{\circ}$ Básico (Comprensión de Lectura, Matemática y Ciencias Naturales y muestral de Educación Física) y II. ${ }^{\circ}$ Medio (Comprensión de Lectura y Matemática). 
LOS SISTEMAS ESTANDARIZADOS DE EVALUACIÓN EN CHILE: PARTICIPACIÓN DE MARIO LEYTON SOTO Y ERIKA HIMMEL KÖNIG JAIME CAICEO ESCUDERO

TIMSS ${ }^{44}$, PISA ${ }^{45}$ y LLECE ${ }^{46}$; al mismo tiempo, inició la evaluación del desempeño docente, debido a que los profesores son uno de los factores que más inciden en el rendimiento de los estudiantes ${ }^{47}$.

Pero, por otra parte las pruebas del SIMCE han permitido constatar algunas tendencias de interés. Dado que sus puntajes están expresados en una escala comparable, se aprecia que las variaciones en el tiempo son menores. Pero su mayor efecto es que el SIMCE ha permitido también confirmar la gran brecha social existente en nuestra educación: por una parte, se observa que las escuelas cuyos/as alumnos/as provienen de familias pobres y con un bajo nivel de educación o de áreas rurales obtienen los peores puntajes.

Fue así como el sIMCE fue objeto de una revisión sistemática por parte de una comisión ad hoc que nombró en 2003 el Ministerio de Educación y nuevamente participó en ella Erika Himmel. Esta comisión, de composición pluralista y con representación de diversos sectores vinculados a la educación, formuló un conjunto de recomendaciones que reafirmaron el rol central del SIMCE como sistema para monitorear los logros de aprendizaje de los estudiantes. Propuso mantener el carácter censal del SIMCE, manteniendo también la tradición de informar sus resultados a nivel agregado, pero ampliar las mediciones, tanto en términos de los contenidos evaluados como de la frecuencia con que se rinden las pruebas. El informe de la comisión puso un especial acento en potenciar el uso del sistema de medición, para lo cual se recomendó perfeccionar la forma en que se informan sus resultados a diferentes usuarios (profesores, padres y opinión pública). Se propuso asimismo que las mediciones incorporen estándares de desempeño que faciliten la comprensión, interpretación y comunicación de los resultados. Finalmente, se sugirió la mantención de las mediciones internacionales para tener resultados que permitan situar a nuestro país en el contexto regional o global y,

44 El nombre deriva del inglés Trends in International Mathematics and Science Study, prueba que se aplica a $4 .^{\circ}$ y $8 .^{\circ}$ Básico a nivel mundial, estudio liderado por la Asociación Internacional para la Evaluación del Logro Educativo, IEA [cfr. CARIOLA, Leonor et al.: «Sistemas de Evaluación como Herramientas de Políticas», Revista Iberoamericana de Evaluación Educativa, I (I) (2008), pp. 64-78: http://www.rinace.net/riee/numeros/voli-numi/arts.pdf. Consultado el II de julio de 20I2].

${ }_{45}$ El nombre deriva del inglés Programme for International Student Assessment; es un proyecto de la Organización para la Cooperación y el Desarrollo Económico (OCDE) que propone obtener información comparable sobre los resultados que alcanzan los estudiantes de is años de edad de los países participantes en las áreas de Lectura, Matemática y Ciencias.

${ }_{46}$ El nombre corresponde al Laboratorio Latinoamericano de la Calidad de la Educación, iniciativa de la unESCO, con su red de sistemas de evaluación de la calidad de la educación en América Latina. Hasta ahora el LLECE ha implementado dos estudios regionales, en los que se han evaluado los logros de aprendizaje de los estudiantes de $3 .^{\circ}$ y $6 .^{\circ}$ Básico, en las áreas de Lenguaje, Matemática y Ciencias Naturales [ $c f r$. Jurado, Fabio: «La Evaluación de la Escritura para la Evaluación de la Lectura», Revista Iberoamericana de Evaluación Educativa, 2 (I) (2009), pp. I30-I46: http://www.rinace.net/riee/numeros/vol2-numi/art7.pdf. Consultado el I2 de julio de 20I2].

47 Hein, Andreas y Taut, Sandy: «El Uso de Información Evaluativa Externa con Fines Formativos: El Caso de Establecimientos Educacionales Chilenos Participantes de SEPA», Revista Iberoamericana de Evaluación Educativa, 3 (2) (2010), pp. 160-181: http://www.rinace.net/riee/numeros /vol3-num2/art9.pdf. Consultado el I2 de julio de 2012. 
LOS SISTEMAS ESTANDARIZADOS DE EVALUACIÓN EN CHILE: PARTICIPACIÓN DE MARIO LEYTON SOTO Y ERIKA HIMMEL KÖNIG JAIME CAICEO ESCUDERO

de este modo, determinar los niveles de desempeño de los estudiantes chilenos respecto de estándares internacionales ${ }^{48}$.

El trabajo de la comisión permitió constatar el importante consenso que hay en nuestro país en torno al valor y credibilidad que posee el simce. Pese a que se trata de un mecanismo de medición desarrollado al interior del Ministerio de Educación, no existen cuestionamientos acerca de su calidad técnica ni respecto de la forma en que el Estado usa y comunica sus resultados.

Los resultados del SIMCE son usados generalmente por el Ministerio de Educación, los municipios y los sostenedores privados como un sistema de incentivo para que los establecimientos educacionales y los docentes mejoren la calidad de los aprendizajes; sin embargo, también es sabido que sus resultados en diversas ocasiones son malinterpretados y sólo sirven como una especie de publicidad que conlleva a cuestionar al sistema educativo vigente.

Recientemente, un grupo encabezado por la Premio Nacional de Educación 20I3, Beatrice Ávalos Davidson, ha criticado fuertemente el que se haya extendido a tantos niveles la aplicación del SIMCE, llevando a los establecimientos educacionales a preocuparse sólo de preparar a sus alumnos para que respondan bien tal instrumento y descuiden la formación más integral de sus alumnos. Nuevamente, aparece la contradicción planteada por Mario Leyton respecto a la PSU; aquí es la misma crítica: se mide sólo conocimiento y no aptitudes para la vida.

\section{Conclusiones}

Al finalizar este trabajo, ha quedado claro el cumplimiento de los objetivos del mismo, puesto que se describió históricamente las tres pruebas que han existido en la historia del Chile Republicano para ingresar a la universidad: Bachillerato, PAA y PSU, como al mismo tiempo los pros y contras de cada sistema y la participación que le correspondió a los dos Premios Nacionales en la gestación de los dos últimos sistemas de selección para proseguir estudios universitarios: Mario Leyton Soto y Erika Himmel König. La misma descripción se realizó respecto al PER y al SIMCE, sistemas de medición del sistema escolar chileno.

El desarrollo del sistema educativo chileno en cuanto a mayor democratización del mismo ha exigido los cambios estudiados. Pero eso mismo, explica las nuevas exigencias respecto a modificar la PSU y el SIMCE. También se ha visto el desarrollo de la evaluación educacional como disciplina científica que presta un servicio para la motivación y ordenamiento de los aprendizajes. Una de las personalidades que ha intervenido en la elaboración de ambas pruebas, Erika Himmel, precisa que es necesario entenderla como evaluación del aprendizaje, «haciendo visible lo invisible»; textualmente la define como:

${ }^{4}$ Gutiérrez, Jaime et al.: «La Evaluación como Camino para el Cambio en la Cultura Pedagógica y el Mejoramiento de la Labor Docente», Revista Iberoamericana de Evaluación Educativa, 5 (2) (2012), pp. 265-283: http://www.rinace.net/riee/numeros/vol5-num2/arti8.pdf. Consultado el II de julio de 2012. 
LOS SISTEMAS ESTANDARIZADOS DE EVALUACIÓN EN CHILE: PARTICIPACIÓN DE MARIO LEYTON SOTO Y ERIKA HIMMEL KÖNIG JAIME CAICEO ESCUDERO

Proceso que lleva a emitir un juicio respecto a uno o más atributos de algo o alguien, fundamentado en información obtenida, procesada y analizada correctamente y contrastada con un referente claramente establecido, sustentado en un marco de referencia valórico y consistente con él, que está encaminado a mejorar los procesos educacionales y que produce efectos educativos en sus participantes, para lo que se apoya en el diálogo y la comprensión ${ }^{49}$.

La aplicación de su concepción, especialmente en las pruebas estandarizadas, no ha tenido el resultado esperado y su concreción no ha estado exenta de críticas; en efecto, Mario Leyton indica que las teorías actuales de educación señalan como importante el desarrollo de las habilidades del pensamiento más que el contenido; este último debe ser sólo un medio para lograr las habilidades; sin embargo, al evaluar por los test, los estudiantes estudian para contestar las preguntas de las pruebas y no para formarse como personas, y agrega:

Esa evaluación sólo alude a lo cognitivo, y deja de lado lo afectivo, la formación integral. O sea la parte econométrica de la evaluación, y no edumétrica. No como la planteaba el profesor Tyler, la evaluación de los objetivos del Currículum que son integrales, sino en el sentido de resultados en base a las preguntas correctamente contestadas. Entonces a final de cuentas, esto es un círculo porque ahora se ha vuelto a eso. Entonces las disciplinas ahora vuelven a ser asignaturas, y el profesor que sabe harto y que dicta clases, no trabaja con los alumnos... Las evaluaciones terminan siendo un fin y no un 'medio para'so.

El debate, sin duda, continuará y nuevos actores aparecerán en escena. Todo ello fruto de la nueva realidad educacional del país, de las nuevas aspiraciones de los estudiantes y del desarrollo científico de la evaluación.

Al parecer, en lo indicado anteriormente, radica la principal causa de la gran polémica que se ha suscitado en los últimos años en Chile con la PSU y, recientemente con el SIMCE, a propósito de la importancia que le ha dado a este último la Agencia de Calidad -nueva instancia de control establecida por ley recientemente- para categorizar los establecimientos educacionales del país e incluso poder cerrarlos.

49 Himmel, Erika et al.: Hacia una Evaluación Educativa. Aprender para evaluar y Evaluar para Aprender, Santiago de Chile, Ministerio de Educación y Facultad de Educación, Pontificia Universidad Católica de Chile (Texto de Perfeccionamiento para Profesores de 6. Año de Educación General Básica), 2000, p. 25.

so Leyton, Mario: op. cit. 